\title{
Letter From the Editor
}

\author{
Meredith Nahm Zozus
}

Keywords: Clinical Data Management

This inaugural issue introduces the Journal of the Society for Clinical Data Management (JSCDM), a peer-reviewed, scholarly journal publishing innovations in data science and informatics and their application in clinical studies. This mission couldn't be timelier. Informatics and statistics have matured as scientific disciplines. Data science, lying at the interface between informatics and statistics, is on the rise. These disciplines are answering the call for new methods. While there are many places to publish these advances, there are few places to report their application toward advancing clinical and translational research. As a result, published work directly relevant to collecting, managing, and using data to plan, conduct, and report clinical studies is sparse. JSCDM fills this gap in the biomedical literature by providing an outlet for publication of advances in the field with an emphasis on their application, improvement, and evaluation in clinical studies.

The primary audience for such work remains the community of clinical and translational research teams collecting and managing data for clinical studies. It has previously been estimated that there are thirty to forty thousand clinical data managers in the United States alone., ${ }^{1,2}$ Additional potential readers include the large community of investigators planning and conducting clinical studies and those in training to become clinical investigators. The target audience for JSCDM also includes those who consume the results of clinical studies, such as research sponsors, care providers, the general public, and regulators as well as those seeking to develop and evaluate

\section{Debu Baruah, MSc IQVIA}

Catherine Craven, PhD, PhD, MA, MLS, FAMIA

Joe R. and Teresa Lozano Long School of Medicine, University of Texas Health Science Center, San Antonio

Imogene Dunn, PhD

Immunovant, Inc.

Kenneth R. Gersing, MD

National Center for Advancing Translational Sciences (NCATS)

\author{
Stacie Grinnon, MS \\ IQVIA
}

new and existing methods for data collection, processing, and management. JSCDM readers will be able to access and search current and back issues through the online, open access journal platform at jscdm.org.

The JSCDM editorial processes have been developed with the intent of meeting the International Committee of Medical Journal Editors (ICMJE) guidelines and National Library Medicine expectations for scholarly contribution to the biomedical literature. As the name suggests, the journal is managed by the Society for Clinical Data Management. There are no costs to authors to publish in JSCDM. As an open access journal, all JSCDM articles are freely available under a Creative Commons Attribution-Non Commercial-Share Alike license (https:// creativecommons.org/licenses). The cost of producing the journal production is offset through advertising.

We at SCDM are excited to announce the distinguished Editorial Board comprised of researchers and professionals engaged in the collection, management, and use of data in clinical studies. Their experience spans both the regulated therapeutic development industry and academia. The founding JSCDM Editorial Board is comprised of the following Distinguished Associate Editors.

We are equally excited to introduce the first issue of JSCDM. This issue contains the long-awaited evidencebased revisions of the Good Clinical Data Management Practices (GCDMP) chapters on Electronic Data Capture (EDC). These chapters, Electronic Data Capture - Selecting an EDC System, by Maxine Pestronk et al., Electronic

Michelle Nusser-Meany, BA

Mutare LifeSciences, Inc.

Derek Petersen, BS

Biogen

Mary Reilly, MBA

Merck \& Co.

Reza Rostami, MBA

Duke Clinical Research Institute, Duke University

Anthony Solomonides, DPhil, FAMIA

NorthShore University Health System 
Rick Ittenbach, PhD, MHS, MEd

Cincinnati Children's Hospital Medical Center

University of Cincinnati College of Medicine

Nidhin Jacob, MTech

Centre for Human Drug Research, The Netherlands

Ioannis Karageorgos, BSN

Bristol-Myers Squibb

Shannon Labout, BA

Data Science Solutions LLC

Data Capture - Study Implementation and Start-up, by David Eade et al., and Electronic Data Capture - Study Conduct, Maintenance, and Closeout, by Olivia Montano, et al., are based on a systematic scoping review of the EDC literature. These reviews identify and synthesize regulation, regulatory guidance, and evidence relevant to practice, and fill gaps with consensus recommendations from the writing groups. As GCDMP chapters, they have additionally undergone public comment periods as well as the peer-review process required of publication in JSCDM. JSCDM continues the practice started in the year 2020 of considering for publication GCDMP chapters. Moving forward, the GCDMP will be managed as a collection accessible from the JSCDM site.

The scoping review for the EDC chapters identified many articles describing the history of development, dissemination, and ultimately adoption of EDC methods and technology, and as such were historically significant. The works of historical relevance were synthesized by Zozus, Sanns, and Eisenstein in the article titled Beyond EDC to document the history of EDC and to extract knowledge relevant to future development and implementation of new technology in clinical research.

The article by Stokeman et al., titled Risk-based Quality Management in CDM, presents a pooled analysis across twenty studies from five therapeutic areas of the impact of both manual queries and automated rule-based queries on data changes. This observational study significantly supports and ads to the limited literature regarding the effectiveness of data cleaning in clinical studies. The authors make recommendations for improving the efficiency of the data cleaning process in clinical studies.

The article by Buckley et al., titled Direct Data Extraction and Exchange of Local Labs for Clinical Research Protocols: A Partnership with Sites, Biopharmaceutical Firms, and Clinical Research Organizations, reports data availability, time, and query-rate metrics from early pilot studies conducted by two pharmaceutical companies of direct extraction of local lab data from Electronic Health Records (EHRs). A recent review by Garza et al. notes the limited literature on the topic, in particular the very limited number of pilots including more than one site. ${ }^{3}$ Buckley et al. make a significant contribution by reporting pilots involving more than one site. In addition, they summarize implementation challenges.

Together, the articles in this inaugural issue exemplify the current state in the management of data from clinical
Hideki Suganami, PhD

Kowa Company Ltd.

Vadim Tantsyura, DrPH

BioMarin Pharmaceutical Inc.

Janet Welsh, CCDMP

Boehringer Ingelheim

Takuhiro Yamaguchi, PhD

Clinical Research Data Center, Tohoku University Hospital

Tohoku University Graduate School of Medicine

studies. On the one hand, establishing evidence-based best practices for routine data collection and processing through web-based EDC, while at the same time pushing beyond EDC to advance data collection, processing, and use in clinical studies by challenging current approaches such as in the Stokeman et al. article, and by undertaking and reporting pilots of new ones as is done by Buckley et al.

This issue represents the culmination of SCDM's commitment to advance clinical data science and informatics in the service of clinical and translational research through promoting and publishing scholarly work in the area, a commitment to promoting and sharing new knowledge through open-access publication, new editorial policies, a distinguished board of Associate Editors, and the selection and implementation of a new online platform for managing the article submission, peerreview, and publication processes. This transformation of SCDM's publication Data Basics to a scholarly journal would not have been possible without the visionary leadership of the 2020 SCDM Board of Trustees and the Publication Committee Chair Claudine Moore, who also served as the Managing Editor through the transition. By instantiating the formal and enduring link between the science behind and the practice of data collection, handling, and use in clinical research, they have done a great service to the clinical research community and consumers of clinical research alike. To them we owe heartfelt thanks and our gratitude.

\section{Competing Interests}

The author has no competing interests to declare.

\section{References}

1. Summary report for: 15-2051.02 - Clinical Data Managers. Department of Labor. onetonline.org. Published 2020. Updated November 17, 2020. Accessed January 25, 2021.

2. Zozus MN, Lazarov A, Smith LR, et al. Analysis of professional competencies for the clinical research data management profession: implications for training and professional certification. J Am Med Inform Assoc. 2017; 24(4): 737-745. DOI: https://doi.org/10.1093/jamia/ocw179

3. Garza M, Myneni S, Nordo A, et al. eSource for Standardized Health Information Exchange in Clinical Research: A Systematic Review. Stud Health Technol Inform. 2019; 257: 115-124. 
How to cite this article: Zozus MN. Letter From the Editor. Journal of the Society for Clinical Data Management. 2021; 1(1): 8, pp. 1-3. DOl: https://doi.org/10.47912/jscdm.44

Submitted: 11 February $2021 \quad$ Accepted: 12 February $2021 \quad$ Published: 08 March 2021

Copyright: ( 2021 SCDM publishes JSCDM content in an open access manner under a Attribution-Non-Commercial-ShareAlike (CC BY-NC-SA) license. This license lets others remix, adapt, and build upon the work non-commercially, as long as they credit SCDM and the author and license their new creations under the identical terms. See https://creativecommons.org/licenses/by-nc-sa/4.0/. 